\title{
Prevención cardiovascular y actitud de cambio frente a los factores de riesgo: un análisis crítico del estado actual
}

\author{
María Teresa Lira $\mathbf{C}^{\mathbf{l a}}$, Sonia Kunstmann $\mathbf{F}^{1}$, \\ Erika Caballero $\mathrm{M}^{2 a}$, Eduardo $\mathrm{G}$ uarda $\mathrm{S}^{1}$, \\ Luis Villarroel $\mathbf{P}^{3 \mathrm{~b}}$, Juan Carlos Molina $\mathrm{Y}^{4}$.

\section{Cardiovascular prevention and attitude of people towards behavior changes: state of the art}

In recent years the main focus of cardiovascular prevention has been to identify people without clinical evidence of coronary disease, but with a high risk of developing a clinical event. Long term follow up studies show that a young person with a high "Relative Risk" of presenting a cardiovascular event becomes an adult with a high "Absolute Risk" of suffering it. The aim of primary prevention is to avoid the appearance of cardiovascular diseases, delaying the development of atherosclerosis and its consequences. In this scenario, the first step is to increase awareness among healthy people of their own cardiovascular risk, enhancing their knowledge of their risk parameter values and generating a correct perception of the risk burden that those values mean. Literature review reveals that significant percentages of healthy individuals are unaware of their own values of blood pressure, total cholesterol and blood glucose. Furthermore, people aware of having abnormal parameters have low treatment compliance rates or evidence inconsistency between knowledge and behavior. This paper reviews educational strategies and other factors that influence this knowledge-behavior gap, such as the stages of behavior changes of the Prochaska and Diclemente Model. Evidence has shown that knowledge about cardiovascular risk factors is not enough to influence behavior and that the degree of preparation of people towards behavior changes is a strong predictor of the success of educational and counseling interventions. Local Chilean data from the RICAR project also shows that the profile of behavior change is different for each modifiable cardiovascular risk factor (Rev Méd Chile 2006; 134: 223-30).

(Key w ords: Cardiovascular diseases; Prevention \& control; Risk factors)

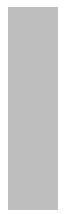

\footnotetext{
Recibido el 29 de septiembre, 2004. Aceptado el 7 de abril, 2005.

${ }^{1}$ Sociedad Chilena de Cardiología y Cirugía Cardiovascular. ${ }^{2}$ Escuela de Enfermería, Universidad Mayor. ${ }^{3}$ Escuela de Salud Pública, Pontificia Universidad Católica de Chile. ${ }^{4}$ Sociedad de Geriatría y Gerontología de Chile.

aEnfermera universitaria

bioestadístico PhD
}

Correspondencia a: María Teresa Lira C. Waterloo 362, Las

Condes. Fono: 2116271. Fax: 6344023. E mail: tlira@puc.cl 
T a enfermedad ateroesclerótica se inicia precozmente alrededor de la segunda década de la vida y provoca la muerte al mayor número de personas en el mundo; 17 millones de muertes anuales ${ }^{1}$.

La prevención cardiovascular primaria ayuda a retardar o evitar su aparición, así como el desarrollo de la enfermedad ateroesclerótica y sus consecuencias. Actualmente, existen múltiples intentos para idear mecanismos que permitan identificar precozmente personas que, sin evidencia clínica de enfermedad coronaria (sanas), desarrollarán enfermedad cardiovascular en el futuro ${ }^{2}$. Ellas requieren intervenciones preventivas, farmacológicas y no farmacológicas agresivas, para evitar que se conviertan en pacientes cardiovasculares mañana»,3,4.

Mantener a los individuos sanos es de extraordinaria importancia para la calidad de vida de las personas, y para la economía de los países. En Chile, las enfermedades cardiovasculares constituyen la tercera causa de invalidez en el sistema previsional actual, lo que implica un alto costopaís, si se piensa que es población laboralmente activa. Esto, sumado a la expectativa de aumento de prevalencia de enfermedad cardiovascular, significará una mayor demanda de recursos en el futuro ${ }^{5}$.

Dado que el origen de la enfermedad coronaria es multifactorial, es importante estimar, en las personas sanas, su riesgo absoluto de sufirila, lo que se define como la probabilidad de desarrollar enfermedad coronaria, evento cardiovascular fatal o no fatal, en un peńodo determinado de tiempo (generalmente en los próximos 10 años) ${ }^{6}$. Se consideran para su cálculo los principales factores de riesgo cardiovascular como: sexo, edad, niveles séricos de colesterol total, diabetes mellitus, presión arterial sistólica y tabaquismo. Existen actualmente tablas de estratificación de riesgo, tanto americanas como europeas, que han ido adaptándose de acuendo a la situación local del país correspondiente ${ }^{7,8}$.

GRADO DE CONOCIMIENTO DE LOS FACTORES DE RIESGO CARDIOVASCULAR EN LA POBLACIÓN GENERAL: REVISIÓN DE LA LTTERATURA

El primer paso fundamental en la prevención de enfermedades cardiovasculares es tener concien- cia de que se está en riesgo ${ }^{9}$. Esto requiere que cada persona conozca sus cifras de parámetros de riesgo y tenga una percepción correcta del riesgo de enfermedad que esos valores significan.

Una encuesta de 10.029 mujeres adultas realizada por la British Heart Foundation, demostró que 4 de cada 5 mujeres nunca habían hablado respecto de enfermedad cardiovascular con su médico o enfermera, pese a constituir su principal causa de muerte. La falta de conocimiento impide la toma de conciencia respecto del riesgo de enfermedad ${ }^{10}$.

La American Heart Association (AHA) inició, hace algunos años, campañas públicas de toma de conciencia. En 1998 ya se había logrado que un porcentaje importante (50-75\%) de las personas atendidas en los sistemas de salud hubieran sido evaluadas para determinar si presentaban o no hipercolesterolemia ${ }^{11}$. Sin embargo, un estudio más reciente respecto a riesgo cardiovascular en 2.000 mujeres americanas, mostró que $75 \%$ de ellas no conocían sus valores de colesterol ${ }^{9}$. Otra investigación nacional del AHA, en mayores de 40 años, encontró que $51 \%$ de los encuestados no conocía su nivel de colesterol plasmático ${ }^{12}$. Esto demuestra el bajo grado de conocimiento que existe sobre algunos factores de riesgo cardiovascular.

Un estudio en Suecia, que incluyó a un tercio de su población, mostró que $95 \%$ de los encuestados se había tomado al menos una vez la presión arterial y $67 \%$ se había medido el colesterol. A $22 \%$ le habrían dicho alguna vez que eran hipertensos, de ellos, $66 \%$ estaba sin tratamiento. Del total de personas diagnosticadas con colesterol elevado sólo $5 \%$ estaba en tratamiento ${ }^{13}$.

Datos del Canadian Heart Health Database muestran que, de las personas en quienes se encontró colesterol elevado, 65\% no conocía su valor de colesterol y sólo $43 \%$ de los que tenían la presión arterial elevada conocían sus cifras tensionales ${ }^{14}$. Otra serie canadiense mostró que $26 \%$ de las personas evaluadas desconocían su condición de hipertensa, de aquellos hipertensos diagnosticados, $42 \%$ estaba en tratamiento en forma correcta, $16 \%$ estaba en tratamiento pero no se controlaba y $16 \%$ sabía que era hipertenso pero no estaba ni en tratamiento ni en control ${ }^{15}$.

En Italia, de un total de 3.400 personas encuestadas, $75 \%$ respondió haberse controlado 
en los 2 años previos la presión arterial y sólo $50 \%$ se había controlado los lípidos plasmáticos y la glicemia en ese período de tiempo. Cincuenta por ciento de los que sabían que eran hipertensos estaban en tratamiento y de los que sabían que tenían hiperlipidemia sólo $20 \%$ estaba en tratamiento 16 .

En nuestro país, el estudio RICAR (Estudio de Prevención de Riesgo Cardiovascular de la Sociedad Chilena de Cardiología) evaluó el grado de conocimiento de los factores de riesgo de 12.500 adultos sanos entre 30 y 80 años. Ellos respondieron si conocían sus propios valores de presión arterial, colesterol total y glicemia ${ }^{17}$. Asimismo, se consultó si sabían el significado de estas cifras, es decir, si eran normales o estaban alteradas. El 67,2\% (8.676) de la población estudiada no conocía sus valores de presión arterial y 77,9\% (10.057) y $83,1 \%$ (10.728) no sabía sus cifras de colesterol total y glicemia, respectivamente, cifras que son aún más elevadas que en otros países $9,12,14$. En aquellas personas que conocían sus cifras, la interpretación de sus propios valores se resume en la Tabla 1, donde destaca que en aquellas personas que conocían su presión arterial, un tercio de ellos sabían que era anormal y en el caso del colesterol, esto ocurría en la mitad de los $\operatorname{casos}^{18}$. Un análisis posterior mostró que, entre quienes refirieron tener valores normales, existía un porcentaje no despreciable de alteración de estos parámetros al compararse el dato reportado con los hallazgos clínicos ${ }^{19}$.

Esta revisión de las publicaciones en diferentes poblaciones muestra que la percepción de riesgo de sufrir un evento cardiovascular y la importancia relativa de los factores de riesgo percibidos por las personas, es insuficiente, con un sesgo optimista por parte del individuo. Existe una gran brecha entre el riesgo percibido y el riesgo objetivamente medido, lo que podría incidir en los aspectos conductuales ${ }^{20}$.

\section{CONOCIMIENTO Y CONDUCTA}

El saber el resultado de las mediciones personales, o tener conocimiento sobre los factores de riesgo cardiovascular, no basta para que la persona se mueva hacia un cambio en la conducta. ¿Qué podría explicar las incongruencias entre conocimiento y conducta?

Aubin, mostró que saber el resultado del chequeo de colesterol plasmático influyó en forma significativa e inmediata en los hábitos alimenticios de las personas. Consultadas a mediano plazo, habían modificado su dieta para reducir su consumo ( $\mathrm{p}<0,0001)$. La reducción del consumo era mayor en las personas con resultados de colesterol plasmático elevado ${ }^{21}$. Paradójicamente, en empleados de un hospital, el conocer su nivel de colesterol no influyó substancialmente en los cambios de la dieta, independiente del nivel de colesterol que les fue encontrado 22 .

Avis, hace una década, estudió en la población general el nivel de conocimiento del riesgo cardiovascular, la autopercepción de salud y la relación entre conocimiento y conducta. La inconsistencia entre conocimiento y conducta era fre-

Tabla 1. Conciencia del significado de sus propios parámetros en aquellos que conocen su valor

\begin{tabular}{|lccc|}
\hline $\begin{array}{l}\text { Parámetros de } \\
\text { Riesgo } \\
(\mathrm{N})\end{array}$ & $\begin{array}{c}\text { Sabe que es } \\
\text { normal }\end{array}$ & $\begin{array}{c}\text { Sabe que } \\
\text { es anormal }\end{array}$ & $\begin{array}{c}\text { No sabe si es } \\
\text { normal o } \\
\text { anormal }\end{array}$ \\
\hline $\begin{array}{l}\text { Presión arterial } \\
(\mathrm{N}=3.780)\end{array}$ & 2.433 & 1.179 & 168 \\
Colesterol total & $64,4 \%$ & $31,2 \%$ & $4,4 \%$ \\
$(\mathrm{~N}=2.554)$ & 1.230 & 1.227 & 97 \\
Glicemia & $48,2 \%$ & $48 \%$ & $3,8 \%$ \\
$(\mathrm{~N}=1.959)$ & 1.567 & 320 & 72 \\
\hline
\end{tabular}


cuente, especialmente en fumadores y personas con sobrepeso. Concluyó, ya en ese entonces, que el conocimiento no necesariamente conducía a tomar una conducta de reducción de riesgo ${ }^{23}$.

En nuestra primera experiencia nacional de estratificación de riesgo cardiovascular, en un grupo de empleados de hospital y familiares de pacientes coronarios en el año 2000, encontramos que con la intervención educativa realizada, un alto porcentaje de las personas logró modificaciones de sus parámetros de riesgo a mediano plazo $^{24}$. En un análisis posterior, fue interesante ver el patrón individual de conducta de cada persona: algunos mejoraron todos sus parámetros de riesgo, otros ninguno. Esta diversidad encontrada, respecto al rol que juega el conocimiento en la conducta adoptada, podría explicarse mejor si se conociera en qué etapa del proceso de cambio de conductas se encontraban las personas en el momento de la intervención.

Existen múltiples estudios controlados y randomizados de educación breve y concisa, entregada por enfermeras, para la modificación de conducta en personas sanas. Ellos muestran que los grupos intervenidos logran mayores cambios de hábitos y conductas respecto al tabaco, ingesta de grasas saturadas y ejercicio regular, y que estos cambios se mantienen a los 4 y 12 meses de seguimiento ${ }^{25}$. Las revisiones sistemáticas y los metaanálisis de estudios randomizados y controlados muestran que las intervenciones multifactoriales personales o familiares, con o sin tratamiento farmacológico, serían las más efectivas para el control de los factores de riesgo y la reducción de la mortalidad, especialmente en grupos de alto riesgo. Un ejemplo de esto son las extensas revisiones de Ebrahim y las de Ketola ${ }^{26,27}$.

Para que estas estrategias sean efectivas debe existir un plan y un objetivo de común acuerdo entre el equipo de salud y el individuo. Levenkron encontró que aun cuando las personas fueran instruidas acerca de la amenaza o relevancia de un determinado factor de riesgo en su salud, no siempre había coincidencia o acuerdo con su tratante en la elección de cuál conducta o factor corregir primero ${ }^{28}$.

Esto es de mucha importancia, por cuanto el paciente debe sentirse partícipe de las decisiones y debe tomar un compromiso de cambio, que le sea factible de realizar.
INTERVENCIÓN EN FUNCIÓN DE LA ETAPA DEL PROCESO DE CAMBIO: EL PRÓXIMO PASO

Una vez realizada la evaluación del niesgo cardiovascular y la categorización del perfil de riesgo de la persona, es necesario elegir la intervención más adecuada para lograr el cambio hacia una conducta saludable ${ }^{29}$. Sin embargo, esto no puede ser un hecho aislado, la etapa del cambio en que está la persona, en relación con una determinada conducta, ha demostrado ser un poderoso factor predictor de éxito o fracaso de las intervenciones que se realicen ${ }^{7}$.

Prochaska y Diclemente, han propuesto un modelo de cambio de conductas con varias etapas, ya que no todas las personas están igualmente preparadas para realizar un cambio de conducta en un momento dado, aun cuando todas hayan sido evaluadas y conozcan sus factores de riesgo ${ }^{7,30-32}$.

\section{Etapas del proceso:}

1. Pre-contemplación: La persona aún no ha empezado a pensar respecto al cambio u otras alternativas. No tiene planificado cambiar sus conductas de riesgo en el futuro mediato (próximos 6 meses).

2. Contemplación: Ha estado reflexionando sobre el cambio y evaluando algunas alternativas. Está consciente de algunos beneficios de este cambio de conducta.

3. Preparación: La persona ha empezado a dar algunos pasos en la dirección del cambio. Busca información a medida que se prepara para realizarlo.

4. Acción: Inició el cambio, y está abocada a mantenerse en esta nueva conducta. Este período dura alrededor de 6 meses, tiempo calculado para considerar como un cambio de conducta real y estable.

5. Mantención: La persona cambió de conducta y sus esfuerzos están dirigidos a adherirse a ella, evitando una recaída. También se identifica en esta etapa, a las personas que han cambiado de conducta hace más 6 meses.

6. Recaída: La persona logró por un período el cambio de conducta pero regresó a alguna etapa previa.

La etapa de contemplación es particularmente importante, ya que en ella las personas están seriamente pensando en realizar el cambio de conducta. Sin 
embargo, a pesar de las intenciones, tienden a quedarse en esta etapa por períodos largos ( 2 años), transformándose en contempladores crónicos ${ }^{7}$. Esto se debe principalmente a que el balance decisional está equilibrado entre los pro y los contra de realizar un cambio ${ }^{8}$. Un porcentaje importante de los fumadores se encuentran frecuentemente en esta etapa. Hay que ser cuidadosos al interpretar resultados de estudios que sólo demuestren actitud de intención (contemplación) de modificar por ejemplo, el consumo de colesterol y de grasas en la dieta $7,33,34$.

La etapa de la acción es especialmente inestable y tiene un gran riesgo de recaídas. Más que en otras etapas, los factores asociados son determinantes del éxito del cambio. Dentro de estos factores asociados están los intrapersonales (percepción de autoeficacia de lo emprendido), interpersonales (apoyo de su red social) y ambiental (ej: exposición inevitable a ambientes de fumadores) ${ }^{7}$.

Las recomendaciones actuales son que las personas que están en las 2 primeras etapas no deberían ingresar a un programa para dejar de fumar, cambiar la dieta o comenzar un programa de ejercicios. En lugar de intervenciones enfocadas a la acción, que podrían terminar en un fracaso que dificulte aún más nuevos intentos, las personas que se encuentran en estas etapas se benefician más con información, motivación, consejo y estimulación de su propia autoeficacia. Esto los ayudará a avanzar a la siguiente etapa (prepa- ración), en la cual el cambio de conducta está siendo considerado en forma concreta, ${ }^{7}, 32$.

Doherty, evaluó el grado de preparación al cambio de grasa en la dieta, actividad física y consumo de tabaco utilizando el modelo descrito, junto a la medición objetiva de sus parámetros de riesgo. El grado de preparación al cambio de varias conductas mostró que habría sinergia entre las estrategias de ayuda y los métodos para identificar personas más respondedoras ${ }^{35}$.

Steptoe, en un estudio randomizado y controlado, comparó el resultado de cambios de conducta a largo y mediano plazo en un grupo que recibió educación para modificar sus conductas basado en este modelo, comparado con un grupo al que se realizó promoción de la salud habitual. El odds ratio de ambos grupos (grupo intervenido vs grupo control) de moverse a etapas de acción/mantención fue de 2,21 vs 1,30 para el consumo de grasas, 1,89 vs 1,07 para el incremento de actividad física y de 1,77 vs 0,76 para dejar de fumar ${ }^{36}$.

El único estudio nacional masivo, en que se ha aplicado este modelo, es el estudio RICAR ${ }^{17}$, en el cual se evaluó la etapa del proceso de cambio de conducta en que se encontraban las personas frente al tabaco, ejercicio, consumo de colesterol y peso.

En ellos el perfil conductual, etapa en el proceso de cambio en que se encontraba el individuo, fue diferente según el factor de riesgo (Tabla 2).

Tabla 2. Perfil conductual de los factores de riesgo cardiovascular modificables en el proyecto RICAR $(n=12.190)$

\begin{tabular}{|lrrcc|}
\hline Etapa del Proceso de & $\begin{array}{c}\text { Cesación } \\
\text { tabaco }\end{array}$ & $\begin{array}{c}\text { Manejo } \\
\text { del peso }\end{array}$ & $\begin{array}{c}\text { Actividad } \\
\text { física }\end{array}$ & $\begin{array}{c}\text { Consumo } \\
\text { colesterol }\end{array}$ \\
\hline Precontemplación & 926 & 3.741 & 1.629 & 7.764 \\
& $12,2 \%$ & $31,1 \%$ & $13,6 \%$ & $64,5 \%$ \\
Contemplación/Preparación & 1.306 & 5.342 & 3.234 & 333 \\
& $17,3 \%$ & $44,5 \%$ & $27 \%$ & $2,8 \%$ \\
Acción & 618 & 1.147 & 2.416 & 2.491 \\
& $8,2 \%$ & $9,5 \%$ & $20,1 \%$ & $20 \%$ \\
Mantención & 2.994 & 438 & 2.146 & 1.448 \\
& $39,6 \%$ & $3,6 \%$ & $17,9 \%$ & $12 \%$ \\
Recaída & 1.717 & 1.348 & 2.574 & $\mathrm{~S} / \mathrm{D}$ \\
\end{tabular}


Fue interesante encontrar que el perfil conductual respecto del tabaco muestra que la mayoría de los fumadores evaluados en este proyecto $(\mathrm{n}=7.564)$ están en la etapa de mantención, es decir, dejaron de fumar y esta conducta ha sido mantenida en forma satisfactoria ${ }^{18}$, lo que es concordante con los datos de prevalencia de las últimas décadas en Chile, en donde el tabaquismo ha disminuido de 46\% en 1990 (Berríos et al) a $41 \%$ en 1999 (Jadue et al) y a 38\% en 2003 (Kunstmann et al) ${ }^{17}$. Esto posiblemente refleje el resultado de las campañas destinadas a la suspensión del tabaquismo. El análisis de este perfil muestra que el énfasis futuro podría enfocarse a evitar las recaídas, que representa a 1 de cada 3 personas que dejaron de fumar.

Diferente es la situación en relación con el manejo del sobrepeso, en que $75 \%$ de nuestra población se encuentra en etapas de pre-contemplación o contemplación, en que aún no toman medidas para su corrección, siendo un factor de riesgo en ascenso en nuestro país, como muestra la última Encuesta Nacional de Salud ${ }^{37}$.

Más alarmante aún fue lo encontrado al investigar el consumo de alimentos ricos en colesterol, en que $2 / 3$ de nuestra población está en etapa pre-contemplativa, es decir, no tienen planificado cambiar su conducta alimentaria, pese a que 53\% de los adultos de entre 30 y 80 años de la Región Metropolitana tienen hipercolesterolemia ${ }^{17}$.

Respecto a actividad física, la distribución en las etapas de cambio fue más homogénea. El perfil conductual relacionado con la actividad física y el peso corporal, sitúan al mayor porcentaje de las personas en las etapas iniciales del proceso de cambio. Esto podría obedecer a que estos aspectos fueron descritos como factores de riesgo cardiovasculares independientes con posterioridad a otros como el tabaco y el colesterol elevado.

\section{Discusión}

Como se aprecia en la revisión de la literatura, el conocimiento es necesario, pero no suficiente para estimular el cambio de conducta, y se requiere un diagnóstico conductual previo para que las intervenciones tengan éxito. El uso de este tipo de análisis y la estratificación conductual orienta asimismo hacia dónde dirigir los esfuerzos en la intervención y como una forma de evaluar su impacto.

El modelo descrito ha demostrado ser útil en diferentes ámbitos de la atención primaria, como lo reporta la Preventive Services Task Force U.S.A $^{38}$, su aplicación también ha sido evaluada como válida y confiable en otros aspectos de promoción y prevención como por ejemplo en la salud dental ${ }^{39}$ y en estudios masivos en la Unión Europea ${ }^{40}$.

En el área de la salud cardiovascular, el desafío para los profesionales de la salud es detectar precozmente individuos en riesgo; desarrollar programas que permitan al sujeto conocer sus factores de riesgo cardiovascular, comprender su significado patológico y el beneficio de adoptar conductas saludables, así como ayudarlo eficazmente a concretar el beneficio.

En el análisis de los datos nacionales, llama la atención el alto porcentaje de personas que no conocen sus valores de presión arterial, colesterol y glicemia, cuando corresponden a evaluaciones frecuentes como por ejemplo la presión arterial en la consulta o la glicemia en los controles de embarazo y en el estudio preoperatorio. Para mejorar esto es necesario que su control sea aún más frecuente y entregar a las personas sus valores, explicándoles si son o no normales; muchas veces sólo le decimos que es normal, pero no le entregamos sus cifras.

Se debe estimular a que en todo contacto que tenga el individuo con el equipo de salud, sean evaluados sus factores de riesgo cardiovasculares modificables tales como presión arterial, glicemia, índice de masa corporal, entre otros. Esto permitiría hacer un diagnóstico precoz y adoptar medidas terapéuticas, lo que es especialmente importante para la hipertensión arterial, en que existe alta prevalencia, bajo diagnóstico y baja adherencia al tratamiento.

Para el éxito de este desafío preventivo se requiere además de la activa participación de los estamentos gubernamentales, de las organizaciones prestadoras de salud públicas y privadas, y de políticas de salud destinadas no sólo a la curación sino también a la prevención de enfermedades crónicas, como la enfermedad cardiovascular. 


\section{REFERENCIAS}

1. Smith SC JR, Greenland P, Grundy SM. Prevention Conference V. Beyond Secondary Prevention: Identifying the High-Risk Patient for Primary Prevention. Executive Summary. Circulation 2000; 101: 111-6.

2. Schnohr P, Jensen JS, Scharling $H$, Nordestgaard BG. Coronary heart disease risk factors ranked by importance for the individual and community. A 21 years follow-up of 12,000 men and women from The Copenhagen City Heart Study. Eur Heart J 2002; 23: 620-6.

3. Bakx JC, Van Den Hoogen HJM, Van Den Bosch WJHM. Development of blood pressure and the incident of hipertension in men and women over a 18-year period; results of the Nijmegen Cohort Study. J Clin Epidemiol 1999; 52: 531-8.

4. WiLSON SL, PoulteR NR. Cardiovascular risk: its assessment in clinical practice. Br J Biomed Sci 2001; 58: 248-51.

5. Rosengren A, Dotevall A, Eriksson H. Optimal risk factors populations: prognosis, prevalence, and secular trends. Eur Heart J 2001; 22: 136-44.

6. Grundy S, Bazzarre F, Cleeman J, D’Agostino R, Hiш M, Houston-Miler N et al. Prevention Conference V: Beyond Secondary Prevention: Identifying the High-Risk Patients for Primary Prevention: Medical Office Assessment: Writing Group I. Circulation 2000; 101: e3.

7. Wood D, De Backer G, Faergeman O, Graham I, Mancia G, Kalevi Pyörälä K. With other members of the Task Force. Task Force Report. Prevention of coronary heart disease in clinical practice. Recommendations of the Second Joint Task Force of European and other Societies on Coronary Prevention. Eur Heart J 1998; 19: 1434-503.

8. Guy de Backer, Chairperson, (ESC); Ettore AmbroSIONI, (ESC); KNUT BoRCH-Johnsen, (EASD, IDFE); Carlos Brotons, (ESGP/FM); Renata Cifkova, (ESC); Jean DaLongevile et al. European Guidelines on Cardiovascular Disease Prevention in Clinical Practice (constituted by representatives of eight societies and by invited experts). Eur Heart J 2003; 24: 1601-10.

9. Mosca L, Robertson RM, HiL M. Trends in awareness and knowledge of heart disease risk and prevention in US women: results of an AHA nationally representative follow-up survey. Circulation 2004; 109: 573-9.
10. JaCKSON G. Women and heart disease: a British Heart Foundation Initiative. Int J Clin Pract 2003; 57: 75.

11. Houston-MiueR N. Achieving Long-Term Cholesterol control: Strategies for Compliance Clinical Practice Guidelines. Nurse Practitioner's Prescribing Reference. Clinical Management of Dyslipidemia. Philips Healthcare Communications, Inc. 1998.

12. Nash IS, Mosca L, Blumenthal RS, Davidson MH, Smith SC JR, PASTernak RC. Contemporary awareness and understanding of cholesterol as a risk factor: results of an American Heart Association national survey. Arch Intern Med 2003; 163: 1597600.

13. Ovhed I, Odeberg $H$, Troein M, Rastam L Awareness and treatment of cardiovascular disease risk factors among middle-aged Swedish men and women. Scand J Prim Health Care 1998; 16: 165-70.

14. Kirkiand SA, Maciean DR, Langilie DB, Joffres MR, MACPHERSON KM, ANDREOU P. Knowledge and awareness of risk factors for cardiovascular disease among Canadians 55 to 74 years of age: results from the Canadian Heart Health Surveys, 19861992. CMAJ 1999; 161(8 Suppl): S10-6.

15. Joffres MR, Hamet P, RABkin SW, Gelskey D, Hogan $\mathrm{K}$, FODOR G. Prevalence, control and awareness of high blood pressure among Canadian adults. Canadian Heart Health Surveys Research Group. CMAJ 1992; 146: 1997-2005.

16. Angelico F, Del Ben M, Francioso S, Hurtova M, Luti A, Bagnara S et al. Attitudes and approach to cardiovascular risk factors in Italy: results of an electronic questionary survey. Public Heath 2003; 117: 242-9.

17. Kunstmann S, Lra MT, Molna JC, Meruane J, Guarda E, Marchant E et al. Riesgo de presentar un evento cardiovascular a 10 años en personas sanas: Proyecto RICAR. Rev Chil Cardiol 2004; 23: 13-20.

18. Lira MT, Kunstmann S, Cabalero E, Vilarroel L. Cardiovascular Risk Factors in Primary Prevention: Knowledge, Awareness, Attitude and Stage of Behavior Change in 12,500 Chilean People. $5^{\text {th }}$ Forum on Quality of Care and Outcomes Research in Cardiovascular Disease and Stroke, American Heart Association May 2004, pg 27 (Abstract) Circulation 2004; 109: 20.

19. Lra MT, Kunstmann S, Cabalero E, Viluaroel L. Accuracy of Self-Reported Prevalence of Cardio- 
vascular Risk Factors in Primary Prevention in the RICAR Project. Scientific Sessions 2004, American Heart Association (Abstract). Circulation 2004 Supl; 110: III-664.

20. Avis NE, Smith KW, McKinlay JB. Accuracy of perceptions of heart attack risk: what influences perceptions and can they be changed? Am J Public Health 1989; 79: 1608-12.

21. Aubin M, Godin G, Vezina L, Maziade J, Desharnais R. Hipercolesterolemia screening. Does knowledge of blood cholesterol level affect dietary fat intake? Can Fam Physician 1998; 44: 1289-97.

22. BARRERE CC. Hospital employee cholesterol screening: modification of dietary behavior. AAOHN J 1994; 42: 261-9.

23. Avis NE, Mc KInLAY JB, Smith KW. Is cardiovascular risk factor knowledge sufficient to influence behavior? Am J Prev Med 1990; 6: 137-44.

24. Lira MT, Rosales J, Ourcileon A, Ferdinand C, Galardo M, Troncoso L et al. Estratificación de riesgo y educación en prevención primaria: modificación de conductas a mediano plazo en grupos de riesgo. (Abstract) Rev Chil Cardiología 2002; 21: 295.

25. Steptoe A, Doherty S, Rink E, KerRy S, Kendrick T, Miton S. Behavioural counseling in general practice for the promotion of healthy behaviour among adults at increased risk of coronary heart disease: randomized trial. BMJ 1999; 319: 943-7.

26. ЕвRAнiм S, Sмтtн GD. Systematic review of randomized controlled trials of multiple risk factor interventions for preventing coronary heart disease. BMJ 1997; 314: 1666-74.

27. Ketola E, Sipila R, MaKeia M. Effectiveness of individual lifestyle interventions in reducing cardiovascular disease and risk factors. Ann Med 2000; 32: 239-51.

28. LeVenkron JC, Greenland P. Patient priorities for behavioral change: selecting from multiple coronary disease factors. J Gen Intern Med 1988; 3: 224-9.

29. Mustone L. Patient Selection for Primary and Secondary Prevention of Dyslipidemia: Clinical
Practice Guidelines. Nurse Practitioner's Prescribing Reference. Clinical Management of Dyslipidemia. Philips Healthcare Comunic, Inc. 1998.

30. Velicer WF, Rossi JS, Diclemente CC, Prochaska JO. A criterion meassurement model for health behavior change. Addict Behav 1996; 21: 555-84.

31. Prochaska JO, Velicer WF. The transtheoretical model of health behavior change. Am J Health Promot 1997; 12: 38-48.

32. O'Connor A, Drake E, Fiset V, Graham I, Laupacis A, Tugwell P. The Ottawa patient decision aids. Eff Clin Pract 1999; 2: 163-70.

33. Snewng A. Lifestyle interventions for lipid management. Nurse Practitioner's Prescribing Reference. Clinical Management of Dyslipidemia. Philips Health Communications Inc. 1998.

34. Soons KR, LtTle DN, Harvey J. Cholesterol screening in the eldery: changing attitudes. Gerontology 1995; 41: 57-62.

35. Doherty SC, Steptoe A, Rink E, Kendrick T, Milton $S$. Readiness to change health behaviours among patients at high risk of cardiovascular disease. J Cardiovasc Risk 1998; 5: 147-53.

36. Steptoe A, KerRY S, RinK E, HiLton S. The impact of behavioral counseling on stage of change in fat intake, physical activity and cigarette smoking in adults at increased risk of coronary heart disease. Am J Public Health 2001; 91: 265-9.

37. Encuesta Nacional de Salud 2003 http:// epi.minsal.cl/epi/html/invest/ENS/ENS.htm

38. US Preventive Services Task Force. Guide to Clinical Services. Ed. Lippincott, Williams \& Wilkins 1996; 618.

39. Tiшis TS, Stach DJ, Cross-Poune GN, Annan SD, Astroth DB, Wolfe P. The transtheoretical model applied to an oral self-care behavioral change: development and testing of instruments for stages of change and decisional balance. Dent Hyg 2003; 77: 16-25.

40. Kearney JM, De Graaf C, Damkjaer S, Engstrom LM. Stages of change towards physical activity in a nationally representative sample in the European Union. Public Health Nutr 1999; 2(1A): 115-24. 